\title{
Perfusion SPECT in patients with suspected pulmonary embolism
}

\author{
Marika Bajc • Massimo Miniati • Jonas Jögi • Paul D. Stein
}

Received: 11 January 2013 /Accepted: 2 April 2013 /Published online: 8 May 2013

(C) Springer-Verlag Berlin Heidelberg 2013

\begin{abstract}
Purpose Ventilation/perfusion tomography (V/P $\left.\mathrm{P}_{\mathrm{SPCT}}\right)$, with new interpretation criteria and newer tracers for ventilation imaging, has markedly improved the diagnostic yield in acute pulmonary embolism (PE). Here, we evaluated the diagnostic performance of perfusion SPECT $\left(\mathrm{P}_{\mathrm{SPECT}}\right)$ without ventilation imaging.

Methods We studied 152 patients with clinically suspected PE who had been examined with both $\mathrm{V} / \mathrm{P}_{\mathrm{SPECT}}$ and multidetector computed tomographic angiography (MDCTA). The diagnosis or exclusion of PE was decided by the referring clinician based on both the $\mathrm{V} / \mathrm{P}_{\mathrm{SPECT}}$ and/or MD-CTA findings in combination with the clinical findings. $\mathrm{P}_{\mathrm{SPECT}}$ images were retrospectively examined by a physician with experience in the interpretation of planar perfusion scans who was blinded to clinical, $\mathrm{V} / \mathrm{P}_{\mathrm{SPECT}}$ and MD-CTA data. $\mathrm{P}_{\text {SPECT }}$ images were interpreted without the aid of chest radiography. All the patients who were deemed to have PE were given anticoagulant therapy.

Results Of the 152 patients, 59 (39\%) received a final diagnosis of PE, and 19 (32\%) had associated cardiopulmonary diseases such as pneumonia, COPD, or left heart failure. $\mathrm{P}_{\mathrm{SPECT}}$ correctly identified $53(90 \%)$ of the 59
\end{abstract}

\author{
M. Bajc $(\varangle) \cdot$ J. Jögi \\ Department of Clinical Physiology, Skåne University Hospital, \\ Lund, Sweden \\ e-mail: marika.bajc@med.lu.se \\ M. Miniati \\ Department of Experimental and Clinical Medicine, \\ School of Medicine, University of Florence, Florence, Italy \\ P. D. Stein \\ Department of Medicine, Wayne State University School \\ of Medicine, Detroit, MI, USA \\ P. D. Stein \\ Department of Research, St Mary Mercy Hospital, \\ Livonia, MI, USA

patients with PE. The specificity was 88 of 93 (95\%). None of the $P_{\text {SPECT }}$ images was rated nondiagnostic. $\mathrm{P}_{\text {SPECT }}$ yielded an overall diagnostic accuracy of $93 \%$ (95\% confidence interval, CI, 87-96\%). At the observed PE prevalence of $39 \%$, the positive and negative predictive values of $\mathrm{P}_{\text {SPECT }}$ were $91 \%$ (95\% CI, 80-97\%) and $94 \%$ (95\% CI, 86-97\%), respectively.

Conclusion In managing critically ill patients, $\mathrm{P}_{\mathrm{SPECT}}$ might be a valid alternative to $\mathrm{V} / \mathrm{P}_{\text {SPECT }}$ or MD-CTA since it was able to identify most patients with PE with a low falsepositive rate and no inconclusive results.

Keywords Perfusion SPECT · Pulmonary embolism . Ventilation/perfusion SPECT · Lung scintigraphy

\section{Introduction}

Pulmonary embolism (PE) is a common but still underdiagnosed condition. In a survey of the relevant literature from 1945 through 2002, PE was unsuspected or undiagnosed ante-mortem in $84 \%$ of patients who had PE discovered at autopsy [1]. Remarkably, $78 \%$ of patients with large or fatal PE were never suspected of having the disease during life [1]. Raising the suspicion of PE is the fundamental step in the diagnostic work-up because it allows selection of patients in whom objective tests are mandatory to confirm or exclude the diagnosis $[2,3]$. Multidetector computed tomographic angiography (MDCTA) is used by many as the first-line imaging technique for suspected PE. Contrast-enhanced CT has changed the practice of medicine, particularly in emergency departments [4], but it has a number of contraindications, and is associated with a substantial radiation burden to the patient [5].

Ventilation/perfusion (V/P) scintigraphy has long been used in the evaluation of suspected PE. In the first 
Prospective Investigation of Pulmonary Embolism Diagnosis (PIOPED I) study, only a minority of patients with established PE had a high probability on the V/P scan (segmental or lobar perfusion defect with normal ventilation). In most patients, V/P yielded inconclusive results [6]. In recent years, the advent of single photon emission $\mathrm{CT}$ (SPECT), new interpretation criteria, and the use of newer tracers for ventilation imaging have markedly improved the diagnostic accuracy of $\mathrm{V} / \mathrm{P}$ scintigraphy in the diagnosis of acute PE. In a recent, broad investigation, a sensitivity of $99 \%$, and a specificity of $98 \%$ were found [7]. In a prospective study published in 1996, planar perfusion scintigraphy (without ventilation imaging) yielded a sensitivity of $86 \%$ and a specificity of $93 \%$ in comparison with pulmonary angiography as the reference diagnostic standard [8]. Similar results were obtained from a retrospective examination of the planar perfusion scans from the PIOPED II study [9].

In the present study, we evaluated the diagnostic performance of $\mathrm{P}_{\mathrm{SPECT}}$ in a sample of 152 patients suspected of having $\mathrm{PE}$.

If $\mathrm{P}_{\mathrm{SPECT}}$ alone had a sensitivity and specificity similar to that of $\mathrm{V} / \mathrm{P}_{\mathrm{SPECT}}$, it could be a valuable alternative in managing critically ill patients and those who might not be able to cooperate due to multiple trauma or neurological disorders.

\section{Patients and methods}

Sample

Over a 1.5-year period, 152 patients with clinically suspected PE were examined with both V/P $\mathrm{P}_{\text {SPCT }}$ and MD-CTA at Skåne University Hospital, Lund, Sweden. The clinical suspicion of PE was based on the presence of relevant symptoms (unexplained dyspnoea, chest pain, fainting or syncope, haemoptysis, palpitations), and the presence of arterial hypoxaemia and/or signs of right ventricular strain on electrocardiography or echocardiography. No formal assessment of the pretest (clinical) probability of PE was made by the attending physicians.

\section{$\mathrm{V} / \mathrm{P}_{\text {SPECT }}$}

$\mathrm{V} / \mathrm{P}_{\text {SPECT }}$ was performed with a large field-of-view dualhead gamma camera with low-energy all-purpose collimators and a $64 \times 64$ matrix used with 128 projections over $360^{\circ}$. While in the supine position, patients inhaled aerosolized ${ }^{99 \mathrm{~m}}$ Tc-pertechnetate (Technegas; Cyclomedica, Lucas Heights, Australia) or ${ }^{99 \mathrm{~m}}$ Tc-DTPA (SmartVent; Diagnostic Imaging, Welford, UK) until about $30 \mathrm{MBq}$ had reached the lung. The acquisition of each projection took $10 \mathrm{~s}$.
Immediately afterwards, and without moving the patient, $120 \mathrm{MBq}{ }^{99 \mathrm{~m}}$ Tc-MAA, (TechneScan LyoMAA; Mallinckrodt Medical) was given intravenously for the perfusion study. This was followed by perfusion tomography in which the acquisition of each projection took $5 \mathrm{~s}$. The patient carefully maintained his/her supine position during the ventilation/perfusion acquisition. Immobilization lasting 20 min was usually well tolerated even by critically ill patients. Iterative reconstruction was performed using ordered-subsets expectation maximization with eight subsets and two iterations. Ventilation background was subtracted from the perfusion tomogram, and a normalized $\mathrm{V} / \mathrm{P}$ image set $\left(\mathrm{V} / \mathrm{P}_{\text {quotient }}\right)$ was calculated. The methodology is described in full elsewhere [7, 10-12].

$\mathrm{V} / \mathrm{P}_{\text {SPECT }}$ diagnostic criteria

In accordance with the European guidelines:

1. No PE: normal perfusion scan or a maximum of one mismatch point (one subsegment).

2. PE: one segmental or two subsegmental mismatches, at least two points.

3. Disorders other than PE: perfusion and ventilation defects showing match or reversed mismatch. The reader reports if the pattern observed is suggestive of other lung diseases such as pneumonia, left heart failure, chronic obstructive pulmonary disease (COPD) or lung cancer. For the sake of this study, these alternative diagnoses are categorized as 'No PE'.

4. Nondiagnostic for PE: complex V/P defects which make the judgement of mismatch impossible or nontypical V/P defects.

In the present study, the $\mathrm{V} / \mathrm{P}_{\mathrm{SPECT}}$ scans were interpreted by one of ten doctors of the Department of Clinical Physiology, Lund, as part of their daily clinical activities. For practical purposes, the extent of PE, as assessed by $\mathrm{V} / \mathrm{P}_{\text {SPECT, }}$ was categorized as small (occlusion less than $20 \%$ of the pulmonary vascular bed), moderate (21-50\%), or large (greater than $50 \%$ ) [13].

$\mathrm{P}_{\text {SPECT }}$ diagnostic criteria

1. No PE: no perfusion defects.

2. PE: single or multiple wedge-shaped perfusion defects, according to the European Association of Nuclear Medicine Guideline criteria, first used in the Prospective Investigative Study on Acute Pulmonary Embolism Diagnosis (PISA-PED) study [8].

3. Disorders other than PE: perfusion defects other than wedge-shaped [8]. They are categorized here as 'No PE'.

$\mathrm{P}_{\text {SPECT }}$ images were examined retrospectively by one of the authors (M.M., of the University of Florence, Italy) with 
long experience in the interpretation of planar perfusion scans. The reviewer was blinded to clinical, $\mathrm{V}_{\mathrm{SPECT}}$, and MD-CTA data. The $\mathrm{P}_{\mathrm{SPECT}}$ images were interpreted without the aid of chest radiography.

\section{Multidetector CT angiography}

Patients were examined with a 16-multidetector CT scanner during a deep-inspiration breath-hold for $10 \mathrm{~s}$. Technical conditions were: $120 \mathrm{kVp}, 190 \mathrm{mAs}$ per slice, $16 \times 1.5 \mathrm{~mm}$ collimator, $0.5 \mathrm{~s}$ rotation time, and 0.938 pitch. The images were acquired in the caudocranial direction in a $512 \times 512$ matrix. Contrast medium (70 ml; Omnipaque; General Electric) was administered intravenously at $4 \mathrm{ml} / \mathrm{s}$. Angiographic criteria for the diagnosis of PE included the identification of an embolus obstructing a vessel, or the outline of an embolus within a vessel.

Final diagnosis

The final diagnosis (PE present or absent) was established by the physician responsible for the patient's care. It was based on the clinical findings in combination with the results of both $\mathrm{V} / \mathrm{P}_{\mathrm{SPECT}}$ and MD-CTA, or compression ultrasonography showing deep vein thrombosis in the patients in whom $\mathrm{V} / \mathrm{P}_{\mathrm{SPECT}}$ or MD-CTA yielded inconclusive results. All the patients who were deemed to have PE received anticoagulant therapy. Anticoagulation was withheld or withdrawn in those who were deemed not to have PE. All the patients were followed for up to 3 months. Outcome measures included hospital readmissions for any cause, recurrent episodes of PE, and death.

\section{Statistical analysis}

Sensitivity is defined as the proportion of tests categorized as positive for PE among the patients who were deemed to have PE by the final clinical judgement. Specificity is the proportion of tests categorized as negative for $\mathrm{PE}$ among the patients who were deemed not to have PE. Accuracy is the sum of true positive and true negative results divided by the total number of patients. Positive predictive value is the proportion of patients with PE among those who had a positive test. Negative predictive value is the proportion of patients without PE among those who had a negative test. According to the binomial distribution, $95 \%$ confidence intervals (CI) were calculated with continuity correction. The agreement between $\mathrm{P}_{\mathrm{SPECT}}$ and $\mathrm{V} / \mathrm{P}_{\mathrm{SPECT}}$, or MD-CTA ratings was tested using the kappa statistic. The statistical analysis was performed with Stata, version 10 (StataCorp, College Station, TX).

\section{Results}

The 152 patients included had a median age of 61 years (interquartile range 40-73 years), 76 (44\%) were men, 97 (64\%) were outpatients at the time of study entry, and 27 $(18 \%)$ had active cancer. The diagnosis or exclusion of PE was based on the congruent results of MD-CTA and V/P $\mathrm{P}_{\text {SPECT }}$ in 88 patients (58\%), MD-CTA in 9 patients $(6 \%), \mathrm{V} / \mathrm{P}_{\text {SPECT }}$ in 53 patients ( $35 \%$ ), and compression ultrasonography in 2 patients (1\%). A final diagnosis of PE was established in 59 ( $39 \%$ ) of the 152 patients, by both MD-CTA and $\mathrm{V} / \mathrm{P}_{\text {SPECT }}$ in 28 patients, by $\mathrm{V} / \mathrm{P}_{\mathrm{SPECT}}$ in 29 patients, and by compression ultrasonography showing deep vein thrombosis in 2 patients with inconclusive MD-CTA or $\mathrm{V} / \mathrm{P}_{\mathrm{SPECT}}$ results. Of the 59 patients with PE, 19 (32\%) had associated cardiopulmonary disorders (pneumonia in 10, left heart failure in 5 and COPD in 2). One patient had PE, lung cancer and COPD, and one PE and lung cancer. The diagnosis of PE was excluded in 93 patients, by both MD-CTA and V/P $\mathrm{P}_{\mathrm{SPECT}}$ in 61 patients, by MD-CTA in 8 patients, and by $\mathrm{V} / \mathrm{P}_{\mathrm{SPECT}}$ in 24 patients. Of these 93 patients, 13 had pneumonia, 10 acute left heart failure, 10 exacerbation of COPD, and 2 lung cancer.

None of the patients, who were deemed not to have PE, developed symptomatic episodes of PE during a 3-month follow-up. None of the patients with established PE had recurrent embolic events during follow-up, and none died from PE. As shown in Table 1, $\mathrm{P}_{\mathrm{SPECT}}$ correctly identified $53(90 \%)$ of the 59 patients with PE. The specificity was 88 of $93(95 \%)$. None of the $\mathrm{P}_{\text {SPECT }}$ images was rated nondiagnostic. $\mathrm{P}_{\mathrm{SPECT}}$ yielded an overall diagnostic accuracy of $93 \%$ (95\% CI 87-96\%). At the observed PE prevalence of $39 \%$, the positive and negative predictive values for $\mathrm{P}_{\text {SPECT }}$ were $91 \%(95 \%$ CI $80-97 \%)$ and $94 \%$ (95\% CI 86-97\%), respectively. Figure 1 shows characteristic $\mathrm{P}_{\mathrm{SPECT}}$ images in a patient with multiple perfusion defects caused by PE. Figure 2 shows a patient with pneumonia who was misinterpreted as having PE on $\mathrm{P}_{\text {SPECT }}$. Figure 3 shows images in a patient with perfusion defects that were interpreted as true-negative for PE.

Table 1 Sensitivity and specificity of $\mathrm{P}_{\mathrm{SPECT}}$ for $\mathrm{PE}$

\begin{tabular}{lcc}
\hline & Number $(\%)$ of patients & $95 \% \mathrm{CI}$ \\
\hline PE present & & \\
True-positive & $53 / 59(90)$ & $80-96$ \\
False-negative & $6 / 59(10)$ & $4-20$ \\
Nondiagnostic & $0 / 59(0)$ & $0-5$ \\
PE absent & & \\
True-negative & $88 / 93(95)$ & $89-98$ \\
False-positive & $5 / 93(5)$ & $2-11$ \\
Nondiagnostic & $0 / 93(0)$ & $0-3$ \\
\hline
\end{tabular}


Fig. $1 \mathrm{P}_{\mathrm{SPECT}}$ in a patient with PE: multiple well-delineated wedge-shaped perfusion defects (arrows) on frontal and sagittal slices

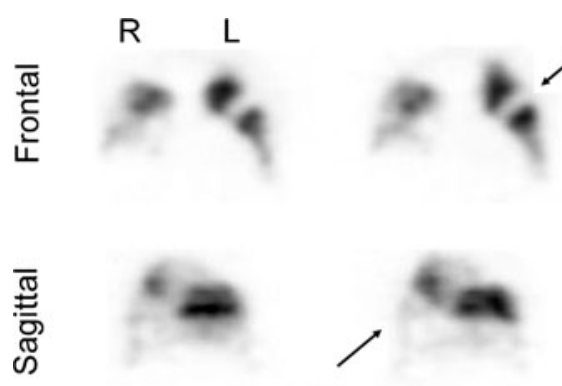

Right lung

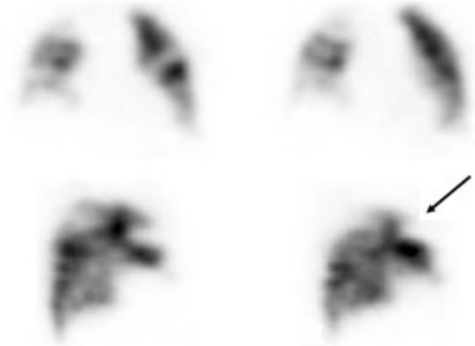

Left lung
The agreement between $\mathrm{P}_{\mathrm{SPECT}}$ ratings and MD-CTA was excellent (observed agreement $98 \%$, kappa 0.95), and it was very good with $\mathrm{V} / \mathrm{P}_{\text {SPECT }}$ (observed agreement $91 \%$, kappa 0.83 ).

\section{Discussion}

PE remains a challenging diagnostic problem because clinicians still fail to raise suspicion of the disease promptly. In recent years, MD-CTA has become the imaging modality of choice for PE in most hospitals worldwide. Mamlouk et al. evaluated retrospectively the medical records of 2003 consecutive patients who underwent MD-CTA for possible PE over a 1.5 -year period [14]. The overall prevalence of PE was only $9.8 \%$ (197/2003), and in particular, the occurrence of a positive angiogram in the patients with no risk factors for $\mathrm{PE}$ was as low as $1 \%(5 / 520)$ [14]. It seems, therefore, that MDCTA is increasingly used as a screening method rather than as a means to confirm or exclude clinically suspected PE. This may contribute to inflating the costs of diagnostic procedures

\section{Sagittal slices}

right lung

\section{Ventilation}
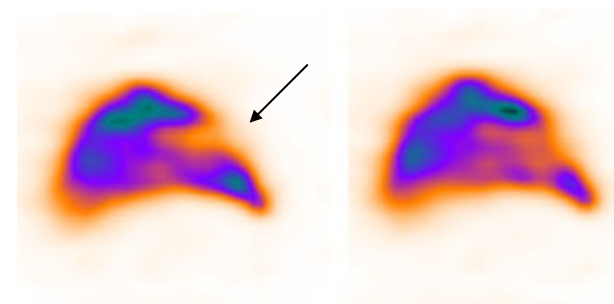

Perfusion
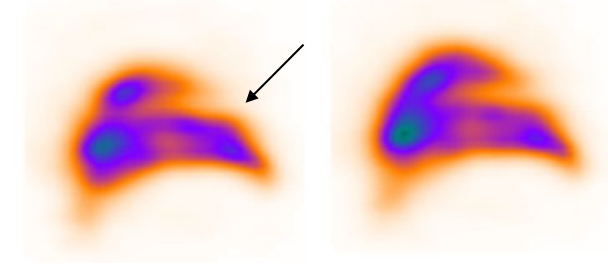

Fig. 2 False-positive $\mathrm{P}_{\mathrm{SPECT}}$ in a patient with pneumonia: single wedgeshaped perfusion defects (arrows) on sagittal slices. Corresponding ventilation images showed a defect in the same area. Laboratory findings and chest radiography were also positive, and the patient was treated for pneumonia for $\mathrm{PE}$, and to expose the patients to an undue amount of radiation. Radiation exposure is of concern, especially in women of child-bearing age. In fact, using a contemporary 64-detector CTA protocol for PE, the absorbed dose to the female breast is in the range 3.5-4.2 cGy [15], which is 30 times greater than that absorbed during $\mathrm{V} / \mathrm{P}$ scintigraphy (0.08 cGy) [10]. In addition, MD-CTA is contraindicated in patients with renal failure, severe heart failure and allergy to contrast agents, and during pregnancy $[10,11]$. Alternative imaging modalities will therefore continue to be needed.

In the present study, using the EANM interpretation criteria, $\mathrm{P}_{\mathrm{SPECT}}$ was accurate in diagnosing or excluding PE with a sensitivity of $90 \%$, and specificity of $95 \%$. In a recent investigation, Gutte et al., interpreted $\mathrm{P}_{\mathrm{SPECT}}$ images in conjunction with low-dose CT using a hybrid gamma camera [11, 16]. They found a sensitivity of $93 \%$ but a specificity of only $51 \%$. Such a low specificity is probably due to the different criteria used for interpreting perfusion defects. In a recent multicentre study using planar imaging and MD-CTA, the sensitivity and specificity for planar lung scintigraphy for the detection of PE were $86 \%$ and $81 \%$, respectively, when PISA-PED interpretation criteria were applied [17]. Sostman et al. found a sensitivity of planar perfusion imaging of $81 \%$ (slightly lower than found be $\mathrm{He}$ at al.), but the specificity was $97 \%[9,17]$. Recently, Morris et al. reported the preliminary clinical application of a new positive tracer for PE consisting of ${ }^{99 \mathrm{~m}} \mathrm{Tc}$-labelled antibodies against the $\mathrm{Fab}^{\prime}$ fragment of the D-dimer moiety $\left({ }^{99 \mathrm{~m}} \mathrm{Tc}-\mathrm{DI}-80 \mathrm{~B} 3\right)$ [18]. They studied 52 patients with moderate or high clinical probability, using MD-

\section{Sagittal slices right lung}

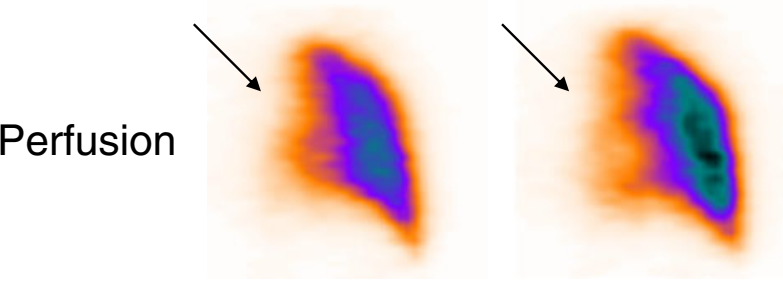

Fig. 3 True-negative $\mathrm{P}_{\mathrm{SPECT}}$ in a patient with left heart failure. Perfusion is redistributed to the anterior parts of the lung (nonsegmental perfusion defect) 
CTA as the reference diagnostic standard. ${ }^{99 \mathrm{~m}} \mathrm{Tc}-\mathrm{DI}-80 \mathrm{~B} 3$ uptake in the lungs was assessed by thoracic SPECT at $2.5 \mathrm{~h}$ after intravenous administration of the labelled antibody. Of 52 the patients, $42(81 \%)$ had both evaluable MD-CTA and a ${ }^{99 \mathrm{~m}} \mathrm{Tc}-\mathrm{DI}-80 \mathrm{~B} 3 \mathrm{scan}$. The latter yielded a sensitivity for PE of $76 \%$ (95\% CI 53-92\%) and a specificity of $90.5 \%$ (95\% CI, $70-99 \%$ [18]. The sensitivity is far lower than ours with $\mathrm{P}_{\text {SPECT. }}$

The high diagnostic accuracy of $\mathrm{P}_{\mathrm{SPECT}}$ is of importance because this technique could be advantageously used in clinical practice, especially in patients in whom ventilation imaging cannot be performed such as those who are clinically unstable or unconscious, or women during the first trimester of pregnancy. However, it should be born in mind that small emboli in the lingula and the right middle lobe might be missed by $\mathrm{P}_{\text {SPECT }}$ due to the shape of these segments. Furthermore, falsepositive $\mathrm{P}_{\mathrm{SPECT}}$ may occur in some patients with pneumonia (Fig. 2) or COPD.

Our study had some limitations. First, in $35 \%$ of the patients, although MD-CTA results were available, the $\mathrm{V} / \mathrm{P}_{\text {SPECT }}$ report was used by the clinicians as referent to diagnose or exclude PE. In some of these patients this was dependent on inconclusive MD-CTA findings. However, it is unlikely that an incorporation bias occurred because the physician in charge of examining the $\mathrm{P}_{\mathrm{SPECT}}$ images was blinded to the reference diagnostic technique and all other clinical data. Second, the study sample was relatively small. Third, $\mathrm{P}_{\mathrm{SPECT}}$ images were interpreted by one reader only. Further studies are needed to assess interobserver agreement in interpreting $\mathrm{P}_{\mathrm{SPECT}}$ images, and to establish whether the examination of chest radiographs could improve the diagnostic performance of $\mathrm{P}_{\mathrm{SPECT}}$ [9]. Fourth, the diagnosis or exclusion of PE was based on the results of two imaging modalities (MD-CTA and V/P $\mathrm{P}_{\mathrm{SPECT}}$ ) that feature different characteristics.

In the past, selective or superselective pulmonary angiography has been used as the reference diagnostic standard for PE $[3,6]$. Conventional pulmonary angiography is now seldom used in clinical practice, its application being restricted to highly specialized centres. In the PIOPED II, a multicentre study aimed at assessing the accuracy of MD-CTA, a composite reference standard was used to diagnose or rule out PE [19]. This included the evaluation of one or more of the following tests: digital subtraction pulmonary angiography (DSA), V/P lung scintigraphy, and venous compression ultrasonography. DSA was performed in only 225 (27\%) of the 824 patients enrolled in the study.

In summary, the present results suggest that $P_{\mathrm{SPECT}}$ might be a valid alternative to $\mathrm{V} / \mathrm{P}_{\text {SPECT }}$ in patients in whom ventilation imaging cannot be performed, since it allows identification of most patients with PE with a low falsepositive rate and no inconclusive results.
Acknowledgments We are grateful to Berit Olsson, research technologist, for invaluable technical assistance.

Conflicts of interest None.

\section{References}

1. Stein PD. Prevalence, risks, and prognosis of pulmonary embolism and deep vein thrombosis. In: Stein PD, editor. Pulmonary embolism. 2nd ed. Oxford: Blackwell; 2007. p. 3-15.

2. Stein PD, Willis III PW, Dalen JE. Importance of clinical assessment in selecting patients for pulmonary arteriography. Am J Cardiol. 1979;43:669-71.

3. Miniati M, Prediletto R, Formichi B, Marini C, Di Ricco G, Tonelli $\mathrm{L}$, et al. Accuracy of clinical assessment in the diagnosis of pulmonary embolism. Am J Respir Crit Care Med. 1999; 159:864-71.

4. Larson DB, Johnson LW, Schnell BM, Salisbury SR, Forman HP. National trends in CT use in the emergency department: 19952007. Radiology. 2011;258:164-73.

5. Smith-Bindman R. Is computed tomography safe? N Engl J Med. 2010;363:1-4.

6. PIOPED Investigators. Value of the ventilation/perfusion scan in acute pulmonary embolism. Results of the prospective investigation of pulmonary embolism diagnosis (PIOPED). JAMA. 1990;263:2753-9.

7. Bajc M, Olsson B, Palmer J, Jonson B. Ventilation/perfusion SPECT for diagnostics of pulmonary embolism in clinical practice. J Intern Med. 2008;264:379-87.

8. Miniati M, Pistolesi M, Marini C, Di Ricco G, Formichi B, Prediletto $R$, et al. Value of perfusion lung scan in the diagnosis of pulmonary embolism. Results of the Prospective Investigative Study of Acute Pulmonary Embolism Diagnosis (PISA-PED). Am J Respir Crit Care Med. 1996;154:138793.

9. Sostman DH, Miniati M, Gottschalk A, Matta S, Stein PD, Pistolesi M. Sensitivity and specificity of perfusion scintigraphy combined with chest radiography for acute pulmonary embolism in PIOPED II. J Nucl Med. 2008;49:1741-8.

10. Bajc M, Neilly JB, Miniati M, Schuemichen C, Meignan M, Jonson B, et al. EANM guidelines for ventilation/perfusion scintigraphy: part 1 . Pulmonary imaging with ventilation/perfusion single photon emission tomography. Eur J Nucl Med Mol Imaging. 2009;36:1356-70.

11. Bajc M, Neilly JB, Miniati M, Schuemichen, Meignan M, Jonson $\mathrm{B}$, et al. EANM guidelines for ventilation/perfusion scintigraphy: part 2. Algorithms and clinical considerations for diagnosis of pulmonary embolism with V/P (SPECT) and MDCT. Eur J Nucl Med Mol Imaging. 2009;36:1528-38.

12. Palmer J, Bitzen U, Jonson B, Bajc M. Comprehensive ventilation/ perfusion SPECT. J Nucl Med. 2001;42:1288-94.

13. Olsson CG, Bitzen U, Olsson B, Magnusson P, Carlsson MS, Jonson B, et al. Outpatient tinzaparin therapy in pulmonary embolism quantified with ventilation/perfusion scintigraphy. Med Sci Monit. 2006;12:PI9-13.

14. Mamlouk MD, van Sonnenberg E, Gosalia R, Drachman D, Gridley D, Zamora JG, et al. Pulmonary embolism at CT angiography: implications for appropriateness, cost, and radiation exposure in 2003 patients. Radiology. 2010;256:62532.

15. Hurwitz LM, Reiman RE, Yoshizumi TT, Goodman PC, Toncheva G, Nguyen G, et al. Radiation dose from contemporary cardiothoracic multidetector $\mathrm{CT}$ protocols with an anthropomorphic female 
phantom: implications for cancer induction. Radiology. 2007;245: $742-50$.

16. Gutte H, Mortensen J, Jensen CV, Bardam Johnbeck C, von der Reck P, Petersen CL, et al. Detection of pulmonary embolism with combined ventilation-perfusion SPECT and low dose CT: head-tohead comparison with multidetector CT angiography. J Nucl Med. 2009;50:1987-92.

17. He J, Wang F, Dai HJ, Li M, Wang Q, Yao Z, et al. Chinese multicenter study of lung scintigraphy and CT pulmonary angiography for the diagnosis of pulmonary embolism. Int $\mathrm{J}$ Cardiovasc Imaging. 2012;28:1799-805.

18. Morris TA, Gerometta M, Yusen RD, White RH, Douketis JD, Kaatz S, et al. Detection of pulmonary emboli with $99 \mathrm{mTc}$-labeled anti-D-dimer (DI-80B3) Fab' fragments (ThromboView). Am J Respir Crit Care Med. 2011;184:708-14.

19. Stein PD, Fowler SE, Goodman LR, Gottschalk A, Hales CA, Hull $\mathrm{RD}$, et al. Multidetector computed tomography for acute pulmonary embolism. N Engl J Med. 2006;354:2317-27. 